\title{
Detritus Type Alters the Outcome of Interspecific Competition Between Aedes aegypti and Aedes albopictus (Diptera: Culicidae)
}

\author{
EBONY G. MURRELL ${ }^{1}$ and STEVEN A. JULIANO \\ Department of Biological Sciences, Illinois State University, Campus Box 4120, Normal, IL 61790
}

\begin{abstract}
Many studies of interspecific competition between Aedes albopictus (Skuse) and Aedes aegypti (L.) (Diptera: Culicidae) larvae show that Ae. albopictus are superior resource competitors to Ae. aegypti. Single-species studies indicate that growth and survival of Ae. albopictus and Ae. aegypti larvae are affected by the type of detritus present in containers, which presumably affects the amount and quality of microorganisms that the mosquito larvae consume. We tested whether different detritus types alter the intensity of larval competition by raising 10 different density/species combinations of Ae. albopictus and Ae. aegypti larvae under standard laboratory conditions, with one of four detritus types (oak, pine, grass, or insect) provided as a nutrient base. Intraspecific competitive effects on survival were present with all detritus types. Ae. albopictus survivorship was unaffected by interspecific competition in all treatments. Negative interspecific effects on Ae. aegypti survivorship were present with three of four detritus types, but absent with grass. Estimated finite rate of increase $\left(\lambda^{\prime}\right)$ was lower with pine detritus than with any other detritus type for both species. Furthermore, Ae. aegypti $\lambda$ ' was negatively affected by high interspecific density in all detritus types except grass. Thus, our experiment confirms competitive asymmetry in favor of Ae. albopictus with oak, pine, or insect detritus, but also demonstrates that certain detritus types may eliminate interspecific competition among the larvae of these species, which may allow for stable coexistence. Such variation in competitive outcome with detritus type may help to account for observed patterns of coexistence/exclusion of Ae. albopictus and Ae. aegypti in the field.
\end{abstract}

\section{Keywords}

Culicidae; Aedes aegypti; Aedes albopictus; condition-specific competition; artificial containers

\begin{abstract}
Interspecific resource competition can be a major determinant of species distributions and ultimately community structure (Connell 1983, Schoener 1983). Often, interspecific resource competition is asymmetrical, which is expected to lead to the competitive exclusion of the species that is more negatively affected by interspecific competition (Lawton and Hassell 1981, Schoener 1983, Vandermeer and Goldberg 2003). However, in some cases, environmental factors may alter the presence or severity of interspecific competition. At the population level, this alteration of competitive effects may reduce asymmetry, or reverse competitive advantage, yielding, respectively, stable coexistence or even a reversal of competitive advantage (Welden and Slauson 1986, Dunson and Travis 1991, Taniguchi and Nakano 2000, Costanzo et al. 2005). This effect is referred to as "condition-specific competition," and it seems to be an important phenomenon in some systems, affecting competitor coexistence, (Taniguchi and Nakano 2000) or local variation in the success and impact of invasive species Facon et al. 2004, Costanzo et al. 2005, Thomas and Holway
\end{abstract}


2005). Most investigations of condition-specific competition have focused on differences in the physical variables that affect competitive interactions (Dunson and Travis 1991, Taniguchi and Nakano 2000, Holway et al. 2002, Facon et al. 2004, Costanzo et al. 2005, Thomas and Holway 2005). Despite its basic and potential practical importance, we know relatively little about the prevalence of condition-specific competition.

A good model system for investigating condition-specific competition is the interaction between two invasive mosquitoes, Aedes albopictus (Skuse) and Aedes aegypti (L.). Ae. albopictus (Diptera: Culicidae) was introduced into Florida in the 1980s, and within a decade had established populations throughout the state (O'Meara et al. 1995). Its spread throughout Florida (Hornby et al. 1994, O'Meara et al. 1995) and southern North America (Hobbs et al. 1991, Mekuria and Hyatt 1995) coincided with the decline of Aedes aegypti, which has inhabited southeastern United States since colonial times (Christophers 1960, Lounibos 2002). Larvae of both species occur in water-filled artificial containers and filter-feed on microorganisms (Juliano et al. 2004, Walker et al. 1996, Merritt et al. 1992), making interspecific resource competition among larvae possible. Laboratory and field studies also indicate that under most conditions Ae. albopictus is the superior competitor (Barrera 1996, Juliano 1998, Daugherty et al. 2000, Braks et al. 2004, Yee et al. 2004), which is consistent with the relative dominance of Ae. albopictus at many Florida sites. Despite this, there are some areas where Ae. aegypti and Ae. albopictus coexist, and in a few areas Ae. aegypti remains dominant and Ae. albopictus has been unable to establish resident populations (O'Meara et al. 1995, Juliano et al. 2004).

The mechanisms producing coexistence of these species at some sites and Ae. aegypti extinction at other sites are unknown. Experiments have shown how a variety of environmental factors may affect the spread of Ae. albopictus (Alto and Juliano 2001a,b), and the pattern of coexistence or exclusion of Ae. aegypti and Ae. albopictus in Florida (Juliano et al. 2002,

Costanzo et al. 2005). Condition-specific competition has been documented for one physical variable (container drying due to drought) in this system (Costanzo et al. 2005). One environmental factor that has received relatively little investigation concerning its effects on competition among mosquitoes is the type of detritus present in containers (Daugherty et al. 2000, Yee et al. 2007a). Different types of detritus support different quantities (and possibly different species) of microorganisms (Walker et al. 1991, Yee and Juliano 2006), which could in turn affect the quantity or quality of food available for mosquito larvae. Differential feeding of mosquito species on microorganism species, or an overabundance of microorganisms available to both species, could lead to elimination or reversal of interspecific competitive advantage between mosquito species, allowing regional coexistence of the two species (Yee et al. 2007a).

Differences in food quality of different detritus types for mosquito larvae have been shown by Daugherty et al. (2000) and Barrera (1996), who demonstrated that larvae raised with animal detritus develop faster and attain larger adult body mass than do larvae raised with plant detritus. Larvae raised with rapidly decaying plant detritus have faster development and larger adult body mass than do larvae raised with slow-decaying plant detritus (Fish and Carpenter 1982, Dieng et al. 2002). Daugherty et al. (2000) showed that addition of insect carcasses to containers primarily based on leaf detritus could eliminate interspecific competition between Ae. Albopictus and Ae. aegypti.

As part of our investigations of the sources of variation in the outcome of competition among these invasive container mosquitoes, we conducted a laboratory experiment on the effects of different detritus types on competition and its population level outcome for Ae. aegypti and Ae. albopictus. Four types of detritus were tested across different density combinations of $A e$. aegypti and Ae. albopictus. We hypothesized that detritus type would alter the presence or 
outcome of interspecific competition, and thus could contribute to observed pattern of coexistence or exclusion in Florida. We expected that reductions of population growth, survival, development, or mass gain due to interspecific competition would be more extreme for Ae. aegypti than for Ae. albopictus in most detritus types. However, we also expected that detritus types that decay rapidly (grass and insect detritus) would reduce or eliminate interspecific competitive effects or reverse competitive asymmetry between these species.

\section{Materials and Methods}

\section{Container Setup}

Forty different treatments were established for this experiment, with three replicates per treatment (120 containers). Each treatment included one of four detritus types-senescent live oak (Quercus virginiana Mill.) leaves; senescent slash pine (Pinus elliotti Engelm.) needles; fresh grass clippings (Zoysia sp.); and insects, consisting of a 50:50 mix, by mass, of Drosophila and crickets (Gryllodes sigillatus) —and one of 10 different density combinations of Ae. albopictus: Ae. aegypti (0:10, 0:20, 0:40, 10:10, 20:20, 10:30, 30:10, 40:0, 20:0, and 10:0), thus testing both intraspecific and interspecific competition at low, medium, and high densities. Larvae were added as first instars, synchronously hatched in $0.44 \mathrm{~g} /$ liter nutrient broth. Detritus types for this experiment were chosen based on the types of detritus commonly found in Florida cemetery vases (S.A.J., unpublished data). With the exception of insect detritus, which was obtained from laboratory colonies, all detritus we used was collected from the Florida Medical Entomology Laboratory, Vero Beach, FL, and it was pesticide-free. Ae. aegypti larvae were from a laboratory colony (generation unknown) originally collected in south Florida. The Ae. albopictus larvae were from an $\mathrm{F}_{2}$ generation colony originally collected at Indrio Rd., Ft. Pierce, FL, and Myakka State Park, Florida.

Each container received $350 \mathrm{ml}$ of deionized (DI) water, $100 \mu \mathrm{l}$ of microbial inoculum of tree hole water collected from Parklands Merwin Preserve, north of Normal, IL, and either $0.5 \pm$ $0.003 \mathrm{~g}$ plant detritus or $0.05 \pm 0.003 \mathrm{~g}$ insect detritus. A lower amount of insect detritus was used because preliminary data indicated that more animal material resulted in fouling of the water and toxicity to larvae. Containers were supplemented with the same amounts of detritus on days 14, 28, and 49 to maintain larval food supply, and DI water was added as needed to maintain the initial volume. The containers were randomized and held in an environmental chamber at $28^{\circ} \mathrm{C}$ on a photoperiod of $14: 10$ (L:D) cycle. When the containers had been established for $4 \mathrm{~d}$ (day 0 of the experiment), we added Ae. albopictus and Ae. aegypti larvae ( $24 \mathrm{~h}$ old) to the containers in the ratios listed above.

Pupae were removed daily and placed in individual vials for eclosion. Eclosed adults were sexed; identified to species; and their date of eclosion, dry mass, and wing lengths of females were recorded. We ended the experiment on day 65 , when we collected and identified remaining larvae. Pupae collected on day 65 were allowed to eclose, and they were included in the adult data set. Once the experiment ended, we calculated survivorship to adulthood for each species in each container.

\section{Population Growth}

We estimated the finite rate of increase $\left(\lambda^{\prime}\right)$ for each species in each container by first calculating the estimated instantaneous rate of increase ( $r^{\prime}$; Livdahl and Sugihara 1984):

$$
\mathrm{r}^{\prime}=\left[\frac{\ln \left[\left(1 / N_{0}\right) \sum_{x} A_{x} f\left(w_{x}\right)\right]}{D+\left[\sum_{x} x A_{x} f\left(w_{x}\right) / \sum_{x} A_{x} f\left(w_{x}\right)\right]}\right]
$$


where $N_{O}$ is the initial number of females per container (assumed to be $50 \%$ of the larvae); $A_{x}$ is the number of females eclosing on day $x$; and $f\left(w_{x}\right)$ is a function describing size dependent fecundity for each species, estimated from the mean wing length on day $x, w_{x}$, of female mosquitoes (Livdahl and Sugihara1984, Juliano 1998). The function for Ae. aegypti was $f$ $\left(w_{x}\right)=2.50 w_{x}-8.616$, where $w_{x}$ is the cube of wing length (millimeters) (Briegel 1990). The function for Ae. albopictus was $f\left(w_{x}\right)=-121.240+78.02 w_{x}$, where $w_{x}$ is wing length (millimeters) (Lounibos et al. 2002). Finite rate of increase was then estimated from $\mathrm{r}$ ' as: $\lambda$ ' $=\exp \left(\mathrm{r}^{\prime}\right)$. For cohort studies like this, $\lambda^{\prime}$ is estimable even if there are no surviving females $\left(\lambda^{\prime}=0\right)$, whereas in that circumstance $r^{\prime}$ is not estimable (Juliano 1998).

\section{Tannins}

In addition to the 120 containers of detritus and mosquitoes larvae, we also established a set of nine containers for each detritus type that did not container larvae. These containers were used to measure decay rate, tannin concentration, and microbial growth rate for each detritus type. Tannin concentrations $>100 \mathrm{mg} / \mathrm{liter}$ can reduce mosquito growth (Sota 1993), and we expected major differences in tannin content among types.

One day before addition of mosquitoes, we collected $10 \mathrm{ml}$ of water from each of the 36 containers that did not receive mosquitoes. These water samples were analyzed for tannins (milligrams per liter) by using a Hach D800 meter and its Hach tannin test kit (Hach, Loveland, $\mathrm{CO})$.

\section{Microbial Growth}

On days 0,4 , and 7 , we collected two 1-ml samples from each of the 36 containers that did not receive mosquitoes. We used these samples to quantify microbial growth using the method of leucine incorporation described by Yee et al. (2007a). Tritiated leucine was added to each sample at a concentration of $25 \mathrm{nM}$. After the samples were incubated for $30 \mathrm{~min}, 100 \%$ trichloroacetic acid was added to each sample (5\% final concentration) to halt leucine incorporation (Yee et al. 2007a). We randomly collected one additional 1-ml sample from two of the 36 containers, and we added trichloroacetic acid to these samples to measure baseline radiation levels of aquatic microorganisms, before the addition of radioactive leucine. All killed samples were then centrifuged, rinsed, and analyzed via liquid scintillation, by using a Beckman LS 6500 scintillation counter (Beckman Coulter, Fullerton, CA). This procedure measures the leucine incorporation into microbial biomass, which quantifies microbial growth (Kirchman 2001).

\section{Detritus Decay Rate}

On days 7,14 , and 21 , we destructively sampled three containers of each detritus type that received no mosquitoes. We used a $106-\mu \mathrm{m}$ sieve to remove remaining detritus from each container, dried the detritus at $50^{\circ} \mathrm{C}$ for at least $24 \mathrm{~h}$, and recorded its dry mass.

\section{Analyses}

For each species we analyzed survivorship and sex-specific mean adult mass and median days to eclosion (collectively "performance variables") by using a linear model (PROC GLM, SAS 9.1), with numbers of Ae. aegypti and Ae. albopictus as continuous variables and detritus type as a class variable. We tested among detritus types for equality of slopes of performance variables versus numbers of the two Aedes species. If detritus type altered the competitive effect of these species on one another or on themselves, we expected that slopes would differ among detritus types, yielding a significant mosquito density $\times$ detritus type interaction. We used factorial analysis of variance (ANOVA) to test effects of Ae. albopictus: Ae. aegypti density combinations on $\lambda^{\prime}$ (which was clearly nonlinearly related to densities), and to test for 
treatment effects on decay rate and tannin concentrations. The effect of detritus type on leucine incorporation rate was analyzed using repeated-measures multivariate analysis of variance (MANOVA) (Scheiner 2001, von Ende 2001). We arcsine square-root-transformed proportions surviving and log transformed mass and time data to meet assumptions of normality and homogeneity of variances. For $\lambda$, data did not meet the assumption of normality, and we also tested for effects using a randomization ANOVA (RT 2.1; Manly 1991). Randomization ANOVA yielded the same significant effects as the parametric ANOVA, hence we report only the parametric results.

\section{Results}

\section{Tannins, Decay Rate, and Microbial Growth}

Tannin concentrations differed significantly among detritus types (Table 1). All detritus types were significantly different from one another, except for pine and insect. Tannins were highest in oak ( $15.2 \mathrm{mg} / \mathrm{liter})$ and lowest in insect $(1.19 \mathrm{mg} / \mathrm{liter})$. Decay rate also differed significantly among detritus types at three times (Table 2), with insect detritus having the greatest percentage of decay, followed by grass (Fig. 1). Oak and pine had low decay, and they were not significantly different from one another. Leucine incorporation differed significantly among detritus types (Table 3). Grass had the highest leucine incorporation, followed by insect. Oak and pine had the lowest incorporation, and they were not significantly different from one another (Fig. 2).

\section{Mosquito Data}

Survivorship for both species differed significantly among detritus types and was negatively affected by conspecific density (Table 4). In addition, there was a significant interaction between heterospecific density and detritus type for Ae. aegypti survivorship, indicating that the impact of interspecific competition differed with detritus type. Heterospecific (Ae. albopictus) density had a significant and negative effect on Ae. aegypti survivorship with pine, oak, and insect detritus, but with grass detritus, survivorship was not significantly affected by Ae. albopictus density (Fig. 3).

Developmental time (Table 5) and mass (Table 6 ) in both species and sexes were significantly affected by both con- and heterospecific densities. Time to eclosion increased significantly and adult mass decreased significantly as larval densities increased. There was a significant interaction of heterospecific density and detritus type for female Ae. albopictus developmental time (Table 5; Fig. 5). Development to adulthood of Ae. albopictus females was less affected by heterospecific density with pine detritus than with other detritus types (Fig. 5B). Also, male Ae. albopictus developmental time showed significant interaction effects between both heteroand conspecific density and detritus type (Table 5), with larval densities having a greater effect on male Ae. albopictus development with pine and insect detritus than with grass and oak detritus (Fig. 5D). There was no interaction for Ae. aegypti males (Fig. 5C). Female masses yielded no significant interactions (Table 6; Fig. 4, A and B). In contrast, male $A e$.

albopictus masses showed a significant interaction between heterospecific density and detritus type (Table 6), with male adult mass of Ae. albopictus more affected by Ae. aegypti density with oak than with other detritus types (Fig. 4D).

Estimated finite rate of increase $\left(\lambda^{\prime}\right)$ of both species was significantly affected by species density combination and detritus type (Table 7). Ae. aegypti showed a significant interaction between combination and detritus type. Specifically, grass detritus yielded significantly higher $\lambda$ ' for Ae. aegypti than did all other detritus types at the 30:10 Ae. albopictus:Ae. aegypti combination (i.e., with high interspecific density), pine yielded significantly lower $\lambda$ ' than did grass at 0:40 combination, and pine also yielded significantly lower $\lambda$ ' than all other detritus 
types at 10:30 and 20:20 combinations (Fig. 6A). For Ae. albopictus, $\lambda$ ' was significantly lower in pine than in all other detritus types, and lower at the 10:10 combination than at all others (Fig. 6B).

\section{Discussion}

It is clear from these data that detritus types not only affect mosquito performance and population growth but also can affect the outcome of competition. The patterns of impacts of larval density on survival and $\lambda$ ' are consistent when compared with the microbial growth and decay rate data across detritus types. Survival and $\lambda^{\prime}$ values for both species were greater with detritus types that had greater decay rates and microbial growth (e.g., grass and insect), and lower with detritus types that had slower decay and microbial growth (e.g., oak and pine). These results suggests that larval survival and $\lambda$ ' are directly related to the amount of available food (microorganisms, as suggested by the microbial growth data) each detritus type supports. These data are consistent with previous laboratory studies of effects of detritus types on mosquito performance (Fish and Carpenter 1982, Walker et al. 1991, Yee and Juliano 2006).

Both the survival and $\lambda^{\prime}$ data support our hypothesis that detritus type can alter the outcome of interspecific competition. The strong negative effect of interspecific competition on Ae. aegypti survival with insect, oak, and pine detritus, with Ae. albopictus simultaneously unaffected by interspecific competition, implies strong competitive asymmetry with $A e$. albopictus the superior competitor with those substrates, which is also consistent with previous studies (Barrera 1996, Juliano 1998, Daugherty et al. 2000, Yee et al. 2004). This competitive asymmetry would be expected to lead to competitive exclusion of Ae. aegypti. However, with grass detritus, neither species' survival was affected by interspecific competition, although intraspecific competition was still evident. Weak interspecific competition along with significant intraspecific competition is consistent with stable coexistence of these two species being possible in containers dominated by grass detritus, as each species' population would be regulated by its own density rather than the density of the other species (Vandermeer and Goldberg 2003). This change from Ae. albopictus dominance to potential coexistence depending on the detritus type could help to explain how these species continue to coexist at some sites in Florida, despite the frequently observed competitive superiority of Ae. albopictus (Barrera 1996, Juliano 1998, Braks et al. 2004). Geographic variation in the mix of detritus types available may affect the outcome of competition, leading to coexistence at some sites and exclusion at others. Sites where coexistence occurs, which are predominantly urban areas of South Florida (O'Meara et al. 1995; S.A.J., unpublished data), would be predicted to have a greater relative abundance of high-quality detritus (e.g., grass), whereas sites dominated by Ae. albopictus would be predicted to have a greater relative abundance of lower quality detritus types (e.g., oak leaves and pine needles).

How may grass as a detritus source yield no interspecific competition but still yield significant intraspecific competition? We cannot answer this question based on this experiment, but we offer a hypothesis: besides producing greater microbial growth (as indicated by leucine incorporation), grass as a resource may yield a greater diversity of microbial types (both taxonomic groups and functional groups), and this diversity may allow these species to specialize on certain classes of microorganisms. Yee et al. (2004) documented significant interspecific differences in foraging behaviors, with Ae. albopictus directing more foraging at detritus surfaces and Ae. aegypti spending more time at the wall and bottom. Under the right environmental circumstances (high microbial abundance and diversity), these differences in foraging behavior may result in differential use of microbial resources, sufficient food for Ae. aegypti (the poorer competitor in many circumstances) and thus reduced interspecific competition. Several other studies suggest that rich, rapidly decaying detritus (usually animal material) can reduce the competitive disadvantage of Ae. aegypti (Barrera 1996, Daugherty et 
al. 2000, Alto et al. 2005). To test this hypothesis, we need data on the diversity of microorganisms associated with grass as a detritus source.

Mass and developmental time of both species showed similar responses to detritus types. This suggests competitive symmetry of the two species for effects on these variables, contrary to our original predictions. However, the profound asymmetry of effects on survival seems to be more important as a determinant of overall population performance $\left(\lambda^{\prime}\right)$. The most consistent and most notable difference between the species is that development time of Ae. albopictus is less affected by interspecific densities in pine detritus than with other detritus types. This response could be in part a result of high mortality of Ae. aegypti with pine detritus (Fig. 3); severe reduction or elimination of Ae. aegypti from high-density pine treatments would alleviate negative interspecific effects on Ae. albopictus, producing the significant interaction effect observed. This asymmetric response in development with pine detritus could provide another mechanism by which Ae. albopictus is competitively superior to Ae. aegypti. However, because survivorship and $\lambda$ ' for both species with pine detritus were very low, it is more likely that this competitive asymmetry serves only to exacerbate competitive dominance of $A e$. albopictus with pine detritus, and it does not ultimately alter the outcome of interspecific competition.

Understanding how environmental factors, such as detritus type, may affect Ae. albopictus and Ae. aegypti distributions is not only of basic ecological interest, but it may be medically important. Both species have been introduced worldwide to tropical and subtropical locations (Hawley 1988), and both are carriers of human diseases such as dengue, yellow fever, and potentially West Nile Virus (Yuill 1986, Sardelis et al. 2002). The ability of both species to live in artificial container habitats (Juliano et al. 2004) brings them into proximity to human population, and along with their ability to transmit human diseases, makes them potentially serious threats to human health on a wide geographic scale. The possibility of stable coexistence between these two vectors under some circumstances raises the question of whether transmission of a disease carried by both (e.g., dengue) might be more frequent or more persistent when these species coexist.

This laboratory experiment provides us with at least one environmental variable, detritus type, that could predict where Ae. aegypti and Ae. albopictus may co-occur, or where Ae.

albopictus may eliminate Ae. aegypti. Logically, the next step should be to determine whether variation in detritus types in nature is correlated with distributions of Ae. aegypti and Ae.

albopictus. Previous studies have shown that Aedes species distributions are correlated with urbanization and climate differences, with Ae. aegypti more prevalent in highly urbanized areas and in locations with greater periods of seasonal drought, whereas Ae. albopictus is highly dominant in rural areas without seasonal drought (Juliano et al. 2002, Braks et al. 2003). We do not yet know urbanization and climate differences are linked to these species distributions. Detritus variation is a possibility, as the quantity and composition of plant and animal communities are generally different along both climatic and urbanization gradients (Mather and Yoshioka 1968, McDonnell and Pickett 1990). Therefore, quanification of geographic variation in detritus types and distributions of these species will be necessary to determine if detritus is in fact an important determinant of Aedes species distributions in the field. Further laboratory or field studies on the chemical and microbial properties of detritus types would help to define the mechanistic relationship between detritus types and their effects on competition.

\section{Acknowledgements}

We thank L. P. Lounibos, B. W. Alto, E. Gunawardene, C. Janiec, W. L. Perry, and S. K. Sakaluk for aid in the field or laboratory, comments, and useful discussion; and three anonymous referees for comments on the manuscript. This 
research was funded by National Institute of Allergy and Infectious Diseases grant R01 AI44793 and by Beta Lambda Chapter of Phi Sigma at Illinois State University.

\section{References Cited}

Alto BW, Juliano SA. Precipitation and temperature effects on populations of Aedes albopictus (Diptera: Culicidae): implications for range expansion. J Med Entomol 2001a;38:646-656. [PubMed: 11580037]

Alto BW, Juliano SA. Temperature effects on the dynamics of Aedes albopictus (Diptera: Culicidae) populations in the laboratory. J Med Entomol 2001b;38:548-556. [PubMed: 11476335]

Alto BW, Lounibos LP, Higgs S, Juliano SA. Larval competition differentially affects arbovirus infection in Aedes mosquitoes. Ecology 2005;86:3279-3288.

Barrera R. Competition and resistance to starvation in larvae of container-inhabiting Aedes mosquitoes. Ecol Entomol 1996;21:112-127.

Braks MAH, Honório NA, Lounibos LP, Oliveira RL, Juliano SA. Interspecific competition between two invasive species of container mosquitoes, Aedes aegypti and Aedes albopictus (Diptera: Culicidae), in Brazil. Ann Entomol Soc Am 2004;97:130-139.

Braks MAH, Honório NA, Oliveira RL, Juliano SA, Lounibos LP. Convergent habitat segregation of Aedes aegypti and Aedes albopictus (Diptera: Culicidae) in southeastern Brazil and Florida, USA. J Med Entomol 2003;40:785-794. [PubMed: 14765654]

Briegel H. Metabolic relationship between female body size, reserves, and fecundity of Aedes aegypti. J Insect Physiol 1990;36:165-172.

Christophers, RS. Aedes aegypti. The yellow fever mosquito: its life history, bionomics, and structure. Cambridge University Press; Cambridge, United Kingdom: 1960.

Connell JH. On the prevalence and relative importance of interspecific competition: evidence from field experiments. Am Nat 1983;122:661-696.

Costanzo KS, Kesavaraju B, Juliano SA. Condition-specific competition in container mosquitoes: the role of non-competing life-history stages. Ecology 2005;86:3289-3295. [PubMed: 17450188]

Daugherty MP, Alto BW, Juliano SA. Invertebrate carcasses as a resource for competing Aedes albopictus and Aedes aegypti (Diptera: Culicidae). J Med Entomol 2000;37:364-372. [PubMed: 15535579]

Dieng H, Mwandawiro C, Boots M, Morales R, Satho T, Nobuko T, Tsuda Y, Takagi M. Leaf litter decay process and the growth performance of Aedes albopictus larvae (Diptera: Culicidae). J Vector Ecol 2002;27:31-38. [PubMed: 12125870]

Dunson WA, Travis J. The role of abiotic factors in community organization. Am Nat 1991;138:10691091.

Facon B, Machline E, Pointier JP, David P. Variation in desiccation tolerance in freshwater snails and its consequences for invasion ability. Biol Invasions 2004;6:283-293.

Fish D, Carpenter SR. Leaf litter and larval mosquito dynamics in tree-hole ecosystems. Ecology 1982;63:283-288.

Hawley WA. The biology of Aedes albopictus. J Am Mosq Control Assoc 1988;1:1-40.

Hobbs JH, Hughes EA, Eichold BH II. Replacement of Aedes aegypti by Aedes albopictus in Mobile, Alabama. J Am Mosq Control Assoc 1991;7:488-489. [PubMed: 1791461]

Hornby JA, Moore DE, Miller TW Jr. Aedes albopictus distribution, abundance, and colonization in Lee County, Florida and its effect on Aedes aegypti. J Am Mosq Control Assoc 1994;10:397-402. [PubMed: 7807083]

Juliano SA. Species introduction and replacement among mosquitoes: interspecific resource competition or apparent competition? Ecology 1998;79:255-268.

Juliano SA, O'Meara GF, Morrill JR, Cutwa MM. Desiccation and thermal tolerance of eggs and the coexistence of competing mosquitoes. Oecologia (Berl) 2002;130:458-469.

Juliano SA, Lounibos LP, O'Meara GF. A field test for competitive effects of Aedes albopictus on Aedes aegypti in south Florida: differences between sites of coexistence and exclusion? Oecologia (Berl) 2004;139:583-593. [PubMed: 15024640] 
Kirchman D. Measuring bacterial biomass production and growth rates from leucine incorporation in natural aquatic environments. Methods Microbiol 2001;30:227-237.

Lawton JH, Hassell MP. Asymmetrical competition in insects. Nature (Lond) 1981;289:793-795.

Livdahl TP, Sugihara G. Non-linear interactions of populations and the importance of estimating per capita rates of change. J Anim Ecol 1984;53:573-580.

Lounibos LP, Saurez S, Menedez Z, Nishimura N, Escher RL, O’Connell SM, Rey JR. Does temperature affect the outcome of larval competition between Aedes aegypti and Aedes albopictus? J Vector Ecol 2002;27:86-95. [PubMed: 12125878]

Lounibos LP. Invasions by insect vectors of human disease. Annu Review Entomol 2002;47:233-266.

Manly, BFJ. Randomization and Monte Carlo methods in biology. Chapman \& Hall; New York: 1991.

Mather JR, Yoshioka GA. The role of climate in the distribution of vegetation. Ann Assoc Am Geogr 1968;58:29-41.

McDonnell MJ, Pickett STA. Ecosystem structure and function along urban-rural gradients: an unexploited opportunity for ecology. Ecology 1990;71:1232-1237.

Mekuria Y, Hyatt MG. Aedes albopictus in South Carolina. J Am Mosq Control Assoc 1995;9:352-355.

Merritt RW, Dadd RH, Walker ED. Feeding behavior, natural food, and nutritional relationships of larval mosquitoes. Annu Rev Entomol 1992;37349(376)

O'Meara GF, Evans LF, Gettman AD, Cuda JP. Spread of Aedes albopictus and decline of Ae. aegypti (Diptera: Culicidae) in Florida. J Med Entomol 1995;32:554-562. [PubMed: 7650719]

Sardelis MR, Turrell MJ, O'Guinn ML, Andre RG, Roberts DR. Vector competence of North American strains of Aedes albopictus for west Nile virus. J Am Mosq Control Assoc 2002;18:284-289. [PubMed: 12542184]

Scheiner, SM. MANOVA: multiple response variables and multispecies interactions. In: Scheiner, SM.; Gurevitch, J., editors. Design and analysis of ecological experiments. 2. chapter 6. Oxford University Press; London, United Kingdom: 2001. p. 99-115.

Schoener TW. Field experiments on interspecific competition. Am Nat 1983;122:240-285.

Sota T. Performance of Aedes albopictus and Aedes riversi larvae (Diptera: Culicidae) in waters that contain tannic acid and decaying leaves: is the treehole species better adapted to treehole water? Ann Entomol Soc Am 1993;86:450-457.

Taniguchi Y, Nakano S. Condition-dependent competition: implications for the distributions of stream fishes. Ecology 2000;81:2027-2039.

Thomas ML, Holway DA. Condition-specific competition between invasive Argentine ants and Australian Iridomyrmex. J Anim Ecol 2005;74:532-542.

Vandermeer, JH.; Goldberg, DE. Population ecology: first principles. Princeton University Press; Princeton, NJ: 2003.

von Ende, CN. Repeated-measures analysis: growth and other time-dependent measures. In: Scheiner, SM.; Gurevitch, J., editors. Design and analysis of ecological experiments. 2. Chapter 8. Oxford University Press; London, United Kingdom: 2001. p. 134-157.

Walker ED, Lawson DL, Merritt RW, Morgan WT, Klug MJ. Nutrient dynamics, bacterial populations, and mosquito productivity in tree hole ecosystems and microcosms. Ecology 1991;72:1629-1546.

Walker ED, O'Meara GF, Morgan WT. Bacterial abundance in larval habitats of Aedes albopictus (Diptera: Culicidae) in a Florida cemetery. J Vector Ecol 1996;21:173-177.

Welden CW, Slauson WL. The intensity of competition versus its importance: an overlooked distinction and some implications. Q Rev Biol 1986;61:23-44. [PubMed: 3961095]

Yee DA, Juliano SA. Consequences of detritus type in an aquatic microsystem: effects on water quality, micro-organisms and performance of the dominant consumer. Freshw Biol 2006;51:448-459. [PubMed: 17476312]

Yee DA, Kesavaraju B, Juliano SA. Interspecific differences in feeding behavior and survival under foodlimited conditions for larval Aedes albopictus and Aedes aegypti (Diptera: Culicidae). Ann Entomol Soc Am 2004;97:720-728.

Yee DA, Kaufman MG, Juliano SA. The significance of ratios of detritus types and microorganism productivity to competitive interactions between aquatic insect detritivores. J Anim Ecol 2007a; 76:1105-1115. [PubMed: 17922707] 
Yuill TM. The ecology of tropical arthropod-borne viruses. Annu Rev Ecol Syst 1986;17:189-219. 


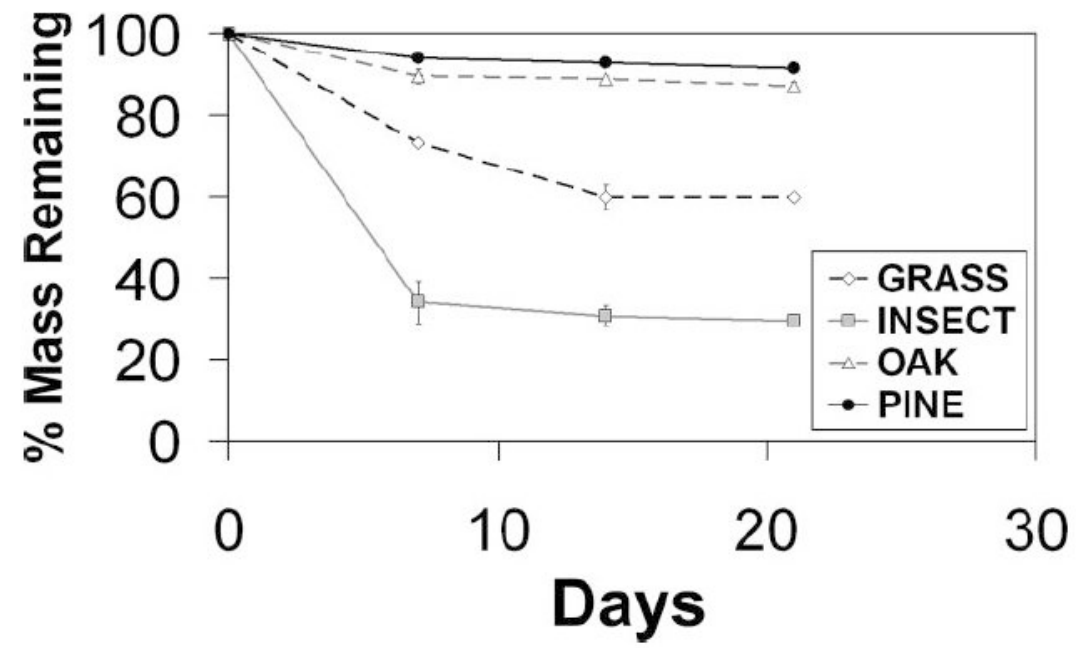

Fig.1.

Decay of different detritus types over time. Values plotted are means \pm SE. Absence of error bars indicates that SE was too small to show. Statistical results in Table 2. 


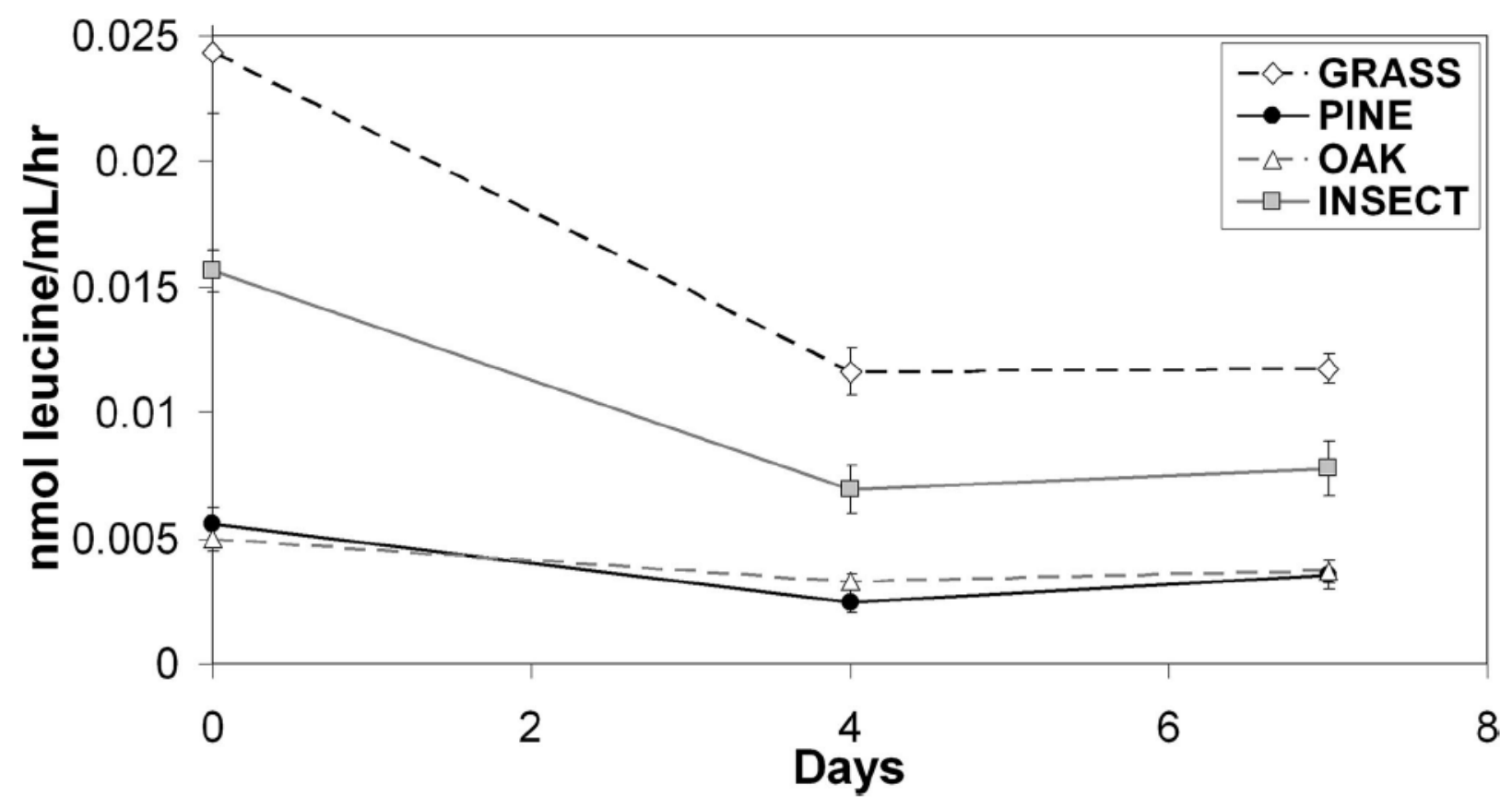

Fig. 2.

Leucine incorporation (mean $\pm \mathrm{SE}$ ) measured at three times for each detritus type. Statistical results in Table 3. 
A

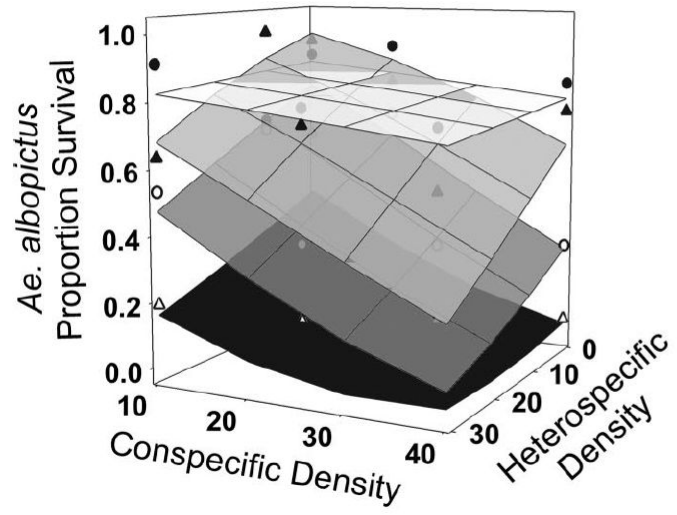

B

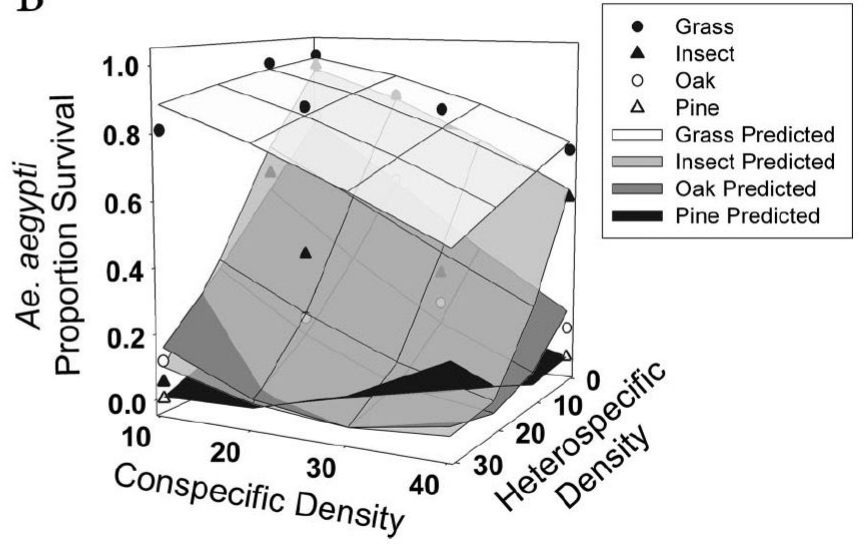

Fig. 3.

Mean proportion survival, by detritus type, of Ae. aegypti (A) and Ae. albopictus (B) as affected by both conspecific and heterospecific densities. Scatter plots represent back-transformed means for each detritus type and surfaces represent predicted model values for each detritus type. Note that appearance of nonlinearity of both scatter plot and surfaces are products of back-transformation. 
A

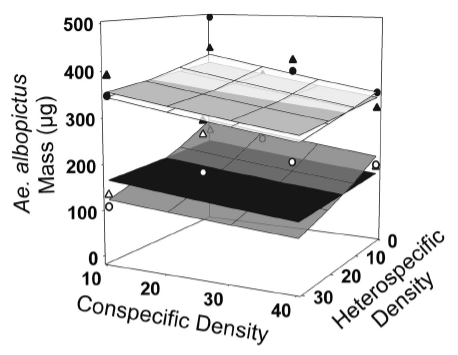

C

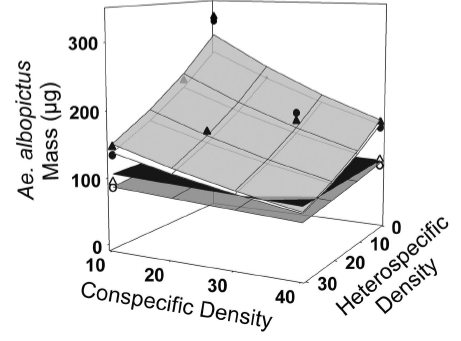

B

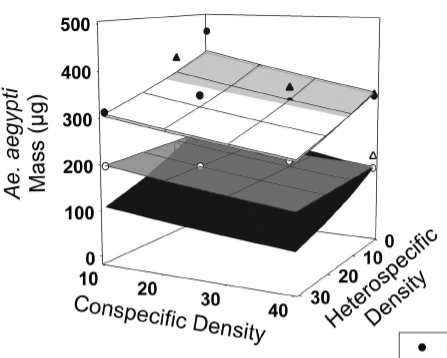

$\mathrm{D}$

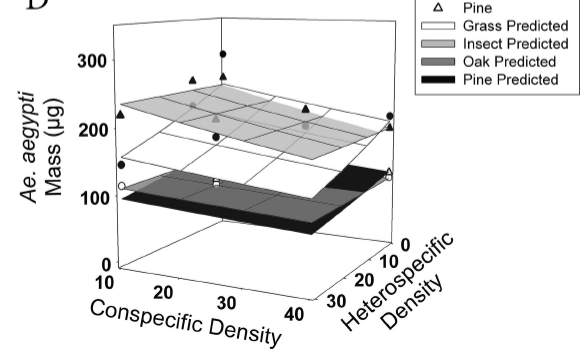

Fig. 4.

Mean mass, by detritus type, of adult females (A and B) and males (C and D) of each species, as affected by conspecific and heterospecific densities. As in Fig. 3, scatter plots represent back-transformed means and surfaces represent predicted model values. Appearance of nonlinearity is a product of back transformation of data. 
A

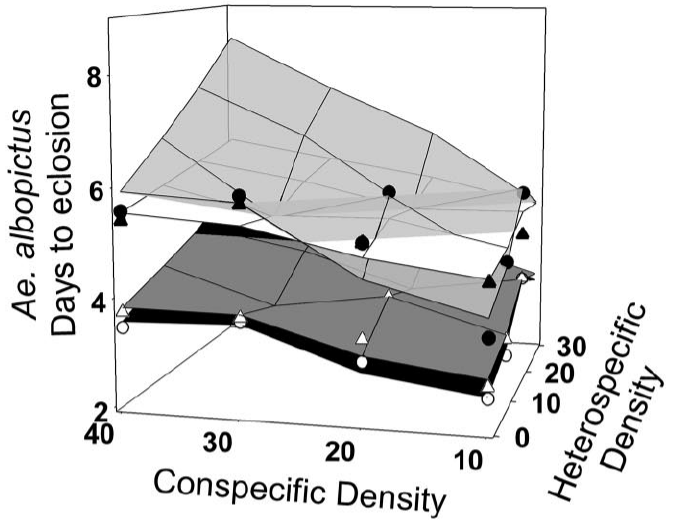

$\mathrm{C}$

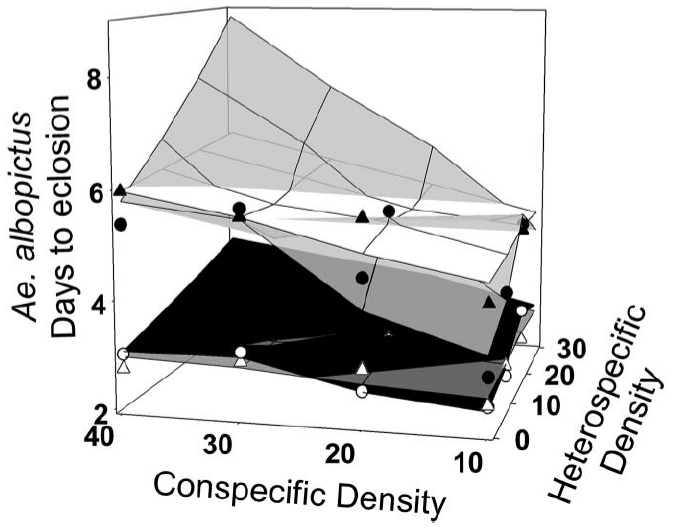

B

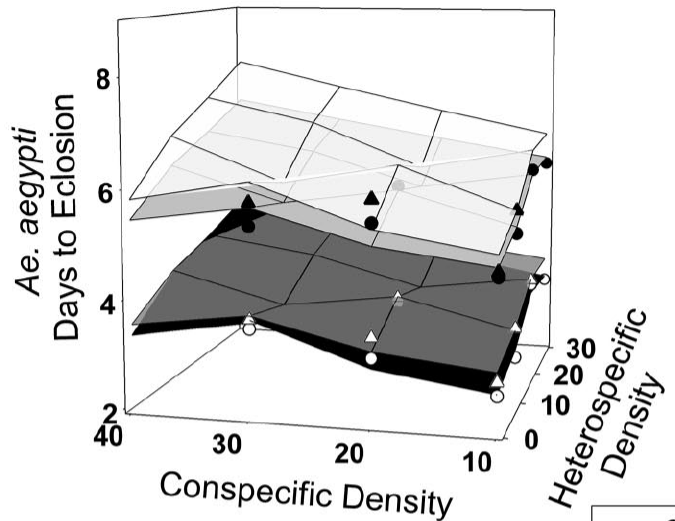

D

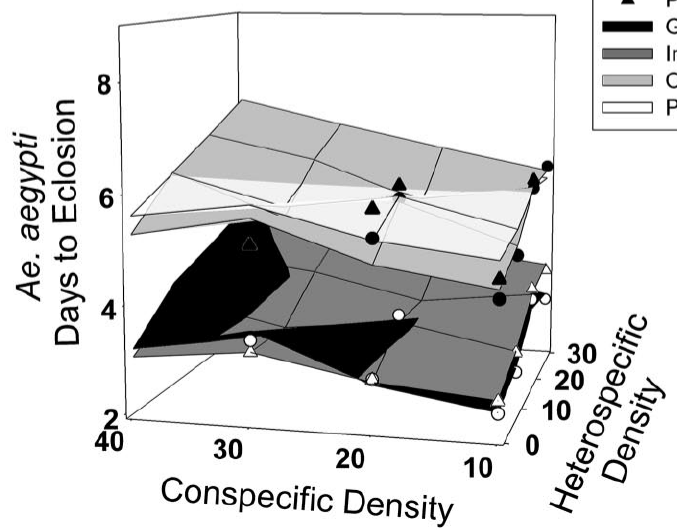

Fig. 5.

Median days to eclosion, by detritus type, of adult females (A and B) and males (C and D) of each species, as affected by conspecific and heterospecific densities. As in Fig. 3, scatter plots represent back-transformed means and surfaces represent predicted model values. Apparent nonlinearity is a product of back-transformation of data. Note that colors for this graph have been reversed to better display all detritus types. 


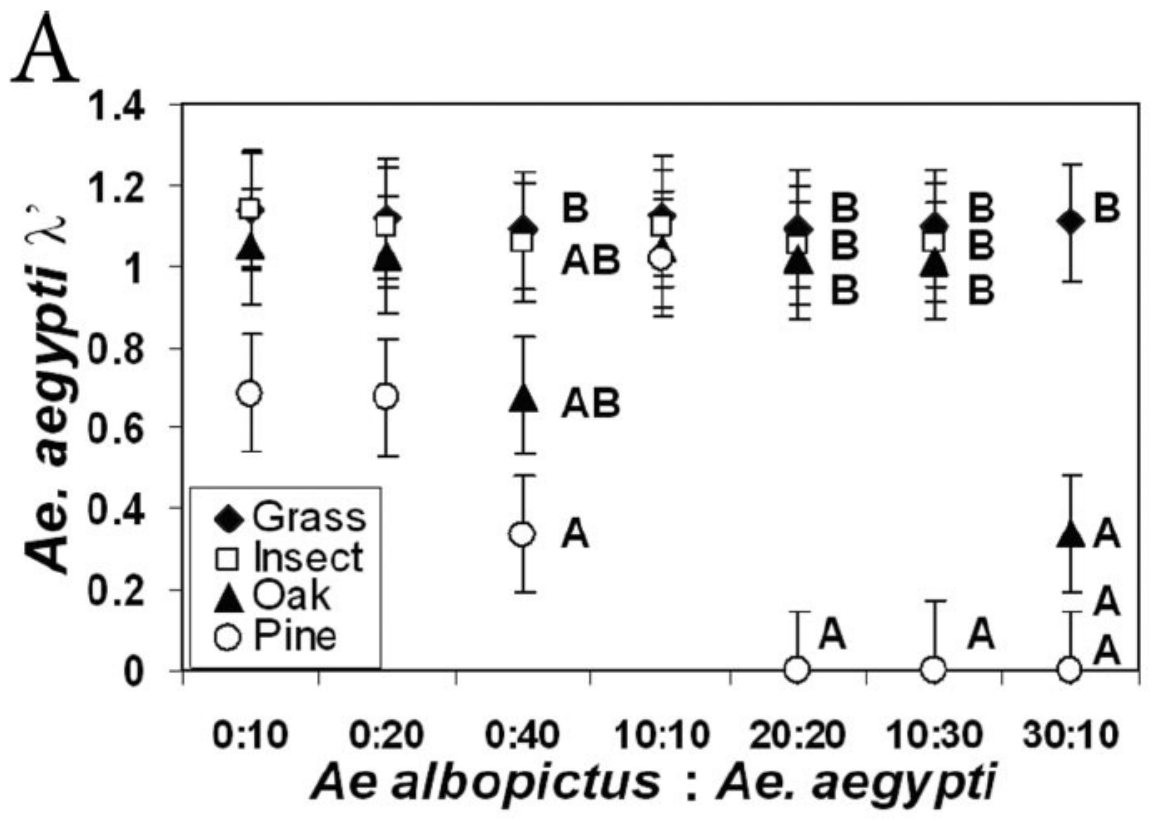

B

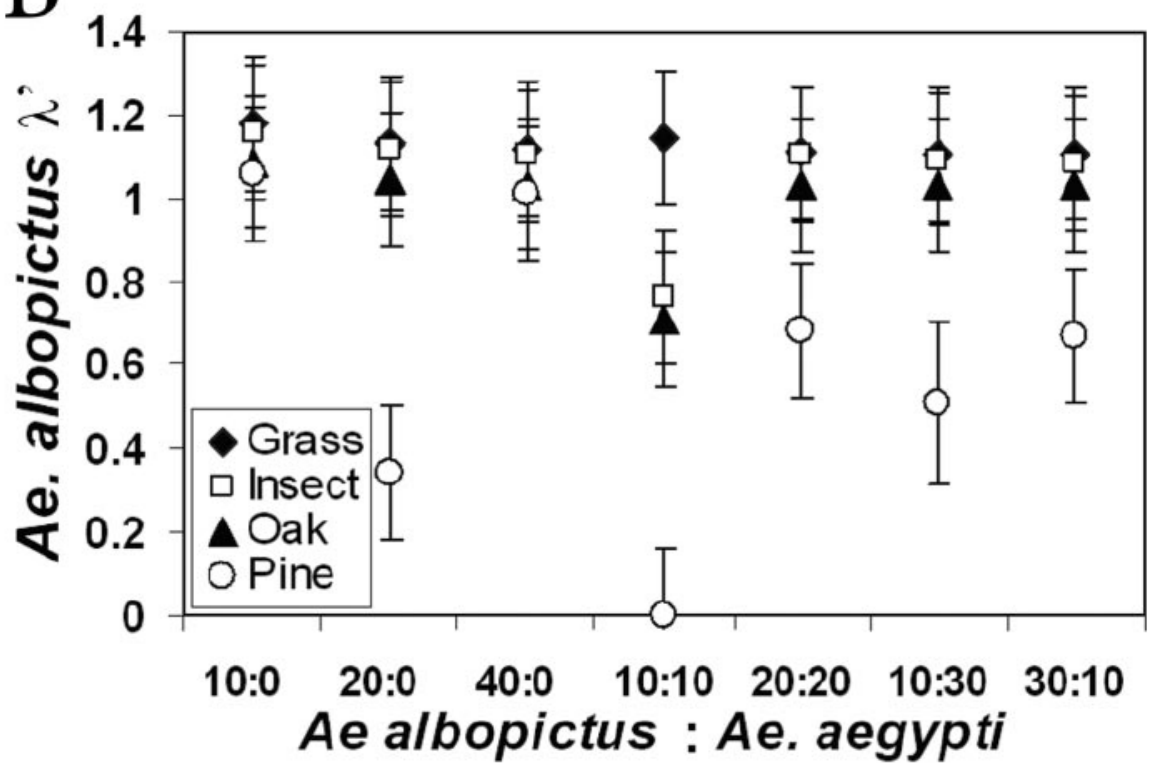

Fig. 6.

Comparison of least squares means for $\lambda$ ' of Ae. aegypti (A) and Ae. albopictus (B). Values with the same letters are not significantly different from one another within the same species combination. 
Table 1

ANOVA and pairwise comparisons for effects of detritus types on tannins

\begin{tabular}{|c|c|c|c|c|}
\hline Source & Comparison & df & $F$ value & $\operatorname{Pr}>F$ \\
\hline Detritus & $\begin{array}{l}\text { Grass vs. insect } \\
\text { Oak vs. pine } \\
\text { Grass vs. oak } \\
\text { Grass vs. pine } \\
\text { Insect vs. oak } \\
\text { Insect vs. pine }\end{array}$ & $\begin{array}{r}3 \\
1 \\
1 \\
1 \\
1 \\
1 \\
1 \\
32\end{array}$ & $\begin{array}{r}73.27 \\
74.27 \\
126.50 \\
19.44 \\
46.76 \\
169.59 \\
3.15\end{array}$ & $\begin{array}{r}<\mathbf{0 . 0 0 0 1} \\
<\mathbf{0 . 0 0 0 1} \\
<\mathbf{0 . 0 0 0 1} \\
\mathbf{0 . 0 0 0 1} \\
<\mathbf{0 . 0 0 0 1} \\
<\mathbf{0 . 0 0 0 1} \\
0.0853\end{array}$ \\
\hline
\end{tabular}

Significant effects and pairwise comparisons are in bold. 
Table 2

ANOVA results for proportion decay of detritus at three time periods: 7, 14, and $21 \mathrm{~d}$

\begin{tabular}{lrrr}
\hline Source & df & $\boldsymbol{F}$ value & $\boldsymbol{P r}>\boldsymbol{F}$ \\
\hline Detritus & 3 & 5652.47 & $<\mathbf{0 . 0 0 0 1}$ \\
Day & 2 & 14.25 & $<.16$ \\
Detritus $\times$ day & 6 & 6.0001 \\
Error & 24 & & $\mathbf{0 . 0 0 0 5}$ \\
& & & \\
\hline
\end{tabular}

Detritus data was square root transformed. Significant $P$ values are in bold. 


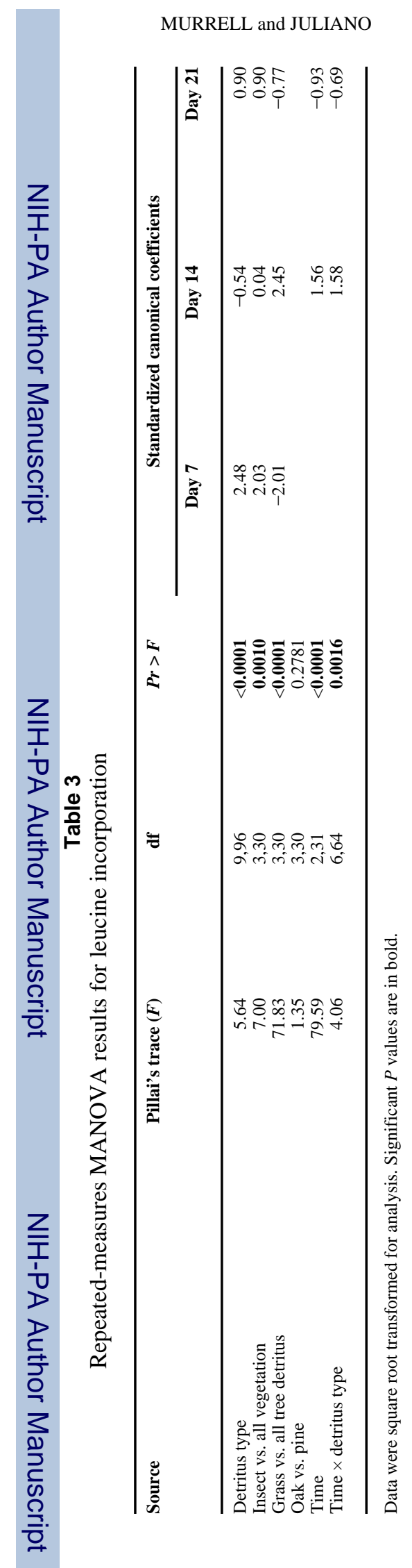

Page 19 


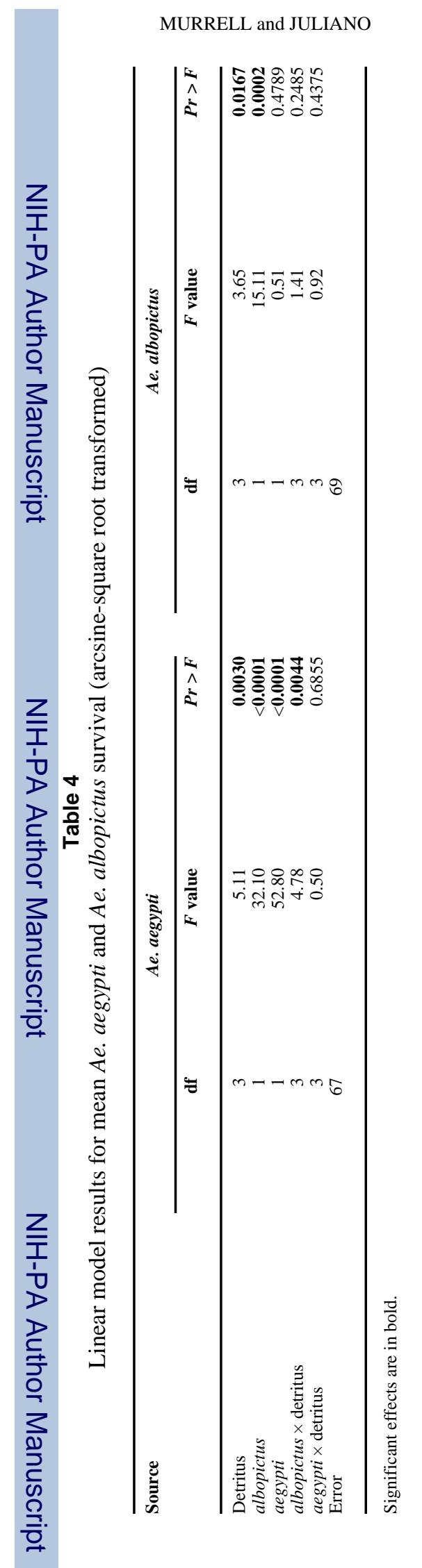

Page 20 


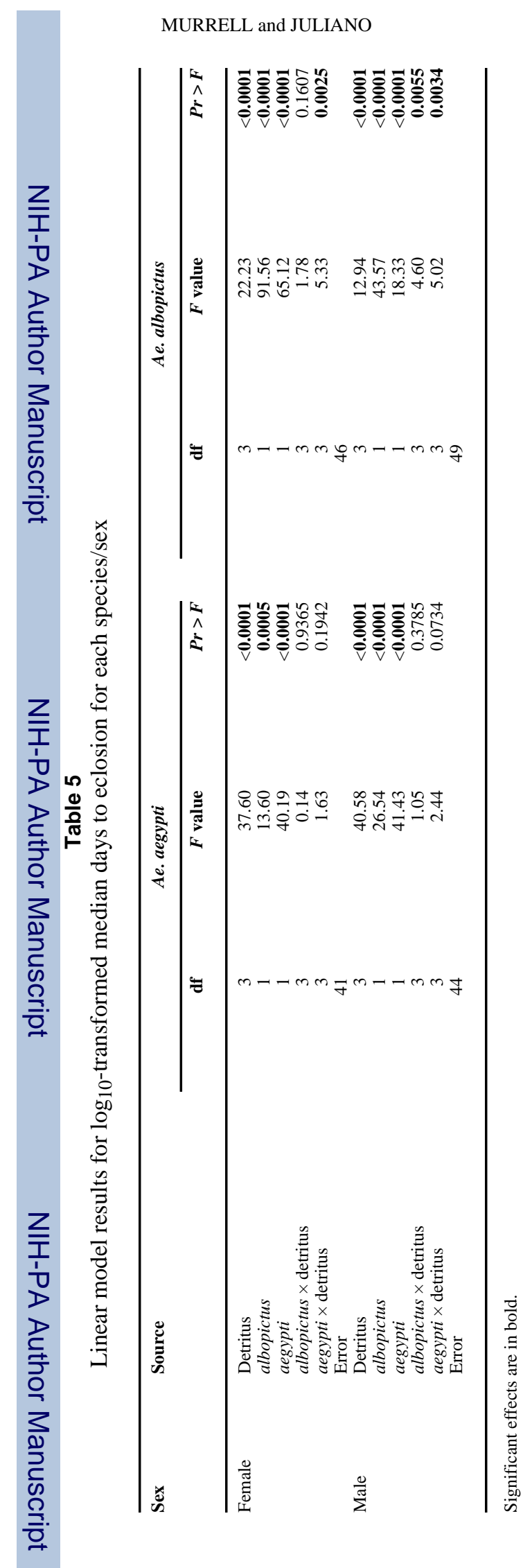

Page 21

J Med Entomol. Author manuscript; available in PMC 2008 November 16. 


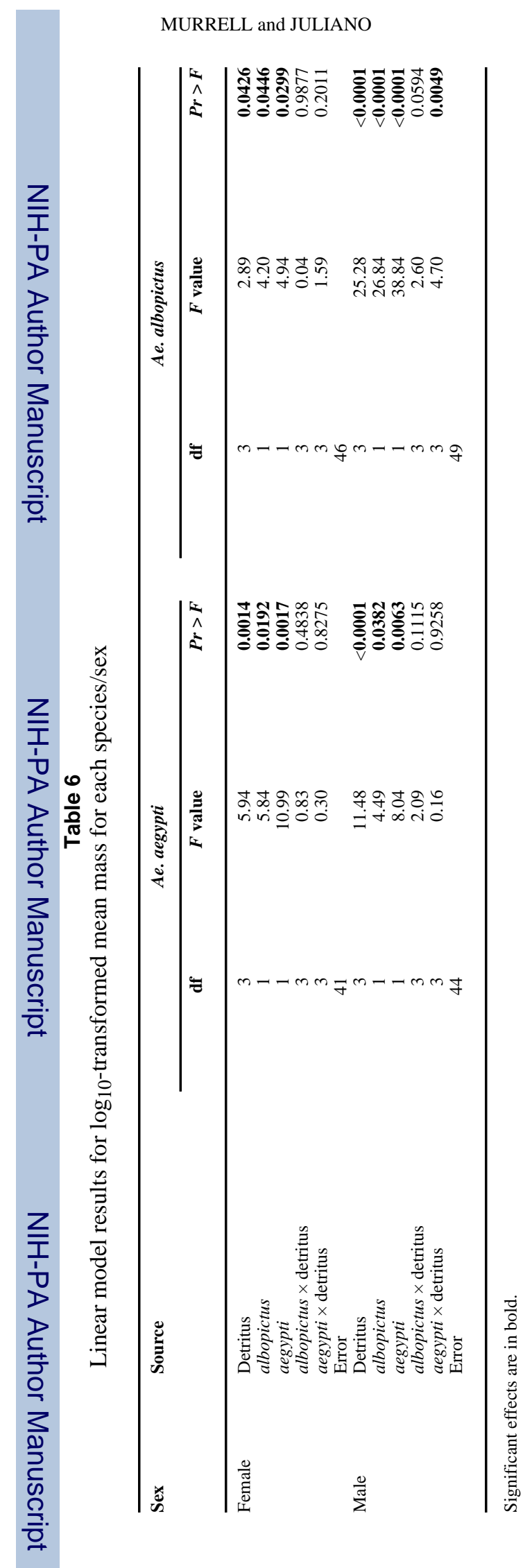

Page 22 


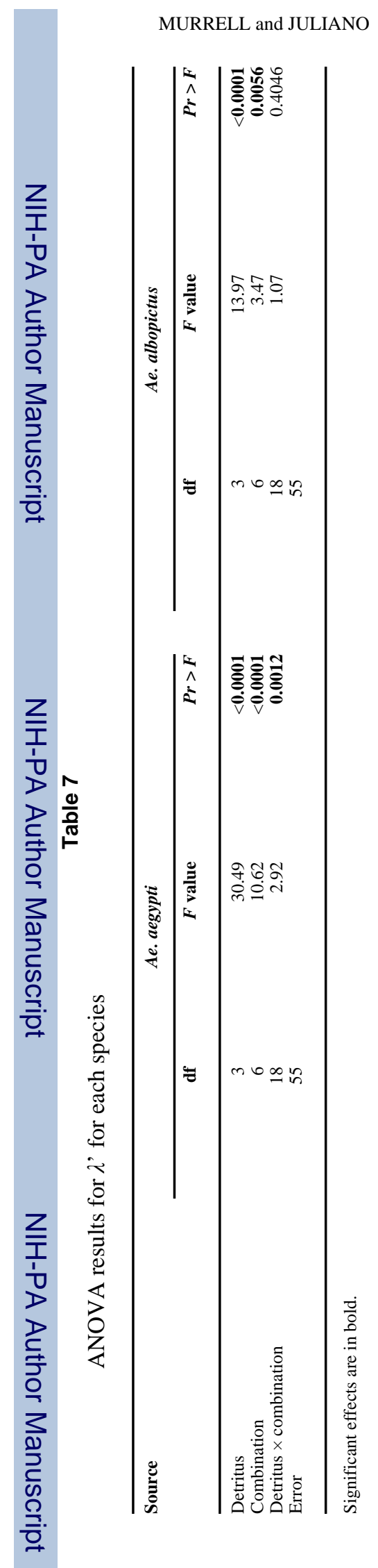

Page 23 